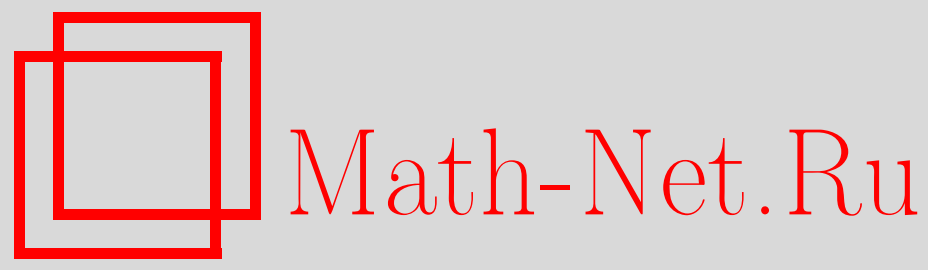

C. В. Нагаев, О больших уклонениях автонормированной суммы, Теория вероятн. и ее примен., 2004, том 49, выпуск 4, 794-802

DOI: https://doi.org/10.4213/tvp197

Использование Общероссийского математического портала Math-Net.Ru подразумевает, что вы прочитали и согласны с пользовательским соглашением

http://www . mathnet.ru/rus/agreement

Параметры загрузки:

IP : 54.162 .85 .209

26 апреля 2023 г., 12:19:50

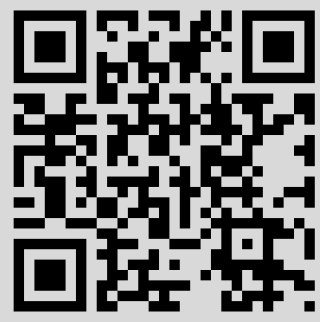


9. Ткачук С. Г. Теорема о больших уклонениях в $\mathbf{R}^{s}$ в случае устойчивого предельного закона. - Случайные процессы и статистические выводы. Ташкент: Фан, 1974 , в. 4 , c. $178-184$.

10. Nagaev $S . V$. Large deviations of sums of independent random variables. - Ann. Probab., 1979, v. 7, № 5, p. 745-789.

Поступила в редакцию 17.III.2003

(c) $2004 \mathrm{r}$.

HAГAEB C. B.*

\section{О БОЛЬШИХ УКЛОНЕНИЯХ АВТОНОРМИРОВАННОЙ СУММЫ ${ }^{1)}$}

В работе выведены экспоненциальные оценки для вероятностей больших уклонений автонормированной суммы независимых случайных величин. При этом не предполагается, что слагаемые одинаково распределены.

Ключевые слова и Фразы: неравенство Берри-Эссеена, отношение Ляпунова, автонормированная сумма, большие уклонения.

1. Введение. Пусть $X_{1}, \ldots, X_{n}$ - независимые случайные величины, $S_{n}=$ $\sum_{i=1}^{n} X_{i}$. Обозначим $b_{j}^{2}=\mathbf{E} X_{j}^{2}, B_{n}^{2}=\sum_{j=1}^{n} b_{j}^{2}, \beta_{3, j}=\mathbf{E}\left|X_{j}\right|^{3}, C_{n}=\sum_{j=1}^{n} \beta_{3, j}$, $L_{n}=C_{n} / B_{n}^{3}$.

Положим $V_{n}^{2}=\sum_{i=1}^{n} X_{i}^{2}$. Целью настояшей работы является изучение больших уклонений автонормированной суммы $S_{n} / V_{n}$, точнее вывод верхних оценок для вероятности $\mathbf{P}\left\{S_{n}>V_{n} x\right\}$ с явно вычисленными постоянными.

Автонормированные суммы изучаются уже давно (см., например, [1]), однако в основном с асимптотической точки зрения и для одинаково распределенных слагаемых (подробную библиографию см., например, в [2]). К.-М. Шао [3] показал, что если $X_{1}, \ldots, X_{n}$ - независимые одинаково распределенные случайные величины с нулевым средним и конечной дисперсией, то для любого $2 \leqslant x \leqslant n^{1 / 2} / 2$

$$
\mathbf{P}\left\{S_{n}>x V_{n}\right\} \leqslant\left(1+x^{-1}\right) \frac{1}{\sqrt{2 \pi} x} \exp \left(-\frac{x^{2}}{2}+A x^{2} \Delta_{n, x}\right),
$$

где $A-$ абсолютная постоянная,

$$
\Delta_{n, x}=\mathbf{E} X^{2} I\left(|X|>\frac{\sqrt{n}}{x}\right)+\frac{x}{\sqrt{n}} \mathbf{E}|X|^{3} I\left(|X| \leqslant \frac{\sqrt{n}}{x}\right) .
$$

Значение постоянной $A$ явно не вычисляется.

К. Ван и Б.-И Джин [4] вывели неравномерную экспоненциальную оценку БерриЭссеена для разности

$$
\delta_{n}(x)=\mathbf{P}\left\{S_{n}<x V_{n}\right\}-\Phi(x),
$$

где $\Phi(x)$ - функция распределения стандартного нормального закона.

С другой стороны, Э. Жине, Ф. Гётце и Д. М. Мейсон [5] доказали, что

$$
\mathbf{E} \exp \left\{\frac{\left(S_{n} / V_{n}\right)^{2}}{4(1+4 e / 3)^{2} M_{n}}\right\} \leqslant 2 \quad \text { и } \quad \mathbf{E} \exp \left\{t\left|\frac{S_{n}}{V_{n}}\right|\right\} \leqslant \exp \left\{\left(1+\frac{4 e}{3}\right) M_{n}^{2} t^{2}\right\}
$$

* Институт математики им. С.Л. Соболева, 630090 Новосибирск-90, Россия; e-mail:nagaev@math.nsk.ru

1) Работа выполнена при поддерже Российского фонда фундаментальных исследований (грант №99-01-00493), а также INTAS (грант № 99-01317). 
для всех $t>0$, где $M_{n}:=\max \left[1, \sup _{k \leqslant n} \mathbf{E}\left|S_{k} / V_{k}\right|\right]$. Второе из этих неравенств используется в [6] при выводе закона повторного логарифма для автонормированных сумм.

Если $X_{i}$ симметричны, то $M_{n}=1$. В обшем случае постоянная $M_{n}$ в работе [5] не оценивается.

Что касается неодинаково распределенных слагаемых, то в работе [7] была получена оценка

$$
\mathbf{P}\left\{S_{n}>x\left(4 B_{n}+V_{n}\right)\right\} \leqslant 24 \exp \left(-\frac{x^{2}}{1152}\right)
$$

в предположении, что $\mathbf{E} X_{j}=0$.

Сформулируем теперь наши основные результаты.

Теорема 1. Пусть $\mathbf{E} X_{i}=0,1 \leqslant i \leqslant n, u C_{n}<\infty$. Тогда для всех $x>0 u$ любого натурального $\mathrm{l}$

$$
\mathbf{P}\left\{S_{n}>x V_{n}\right\}<\left(\frac{x^{2}}{4 \ln 2}+l\right) \exp \left(-\frac{x^{2}}{4}\left(1-2^{3 l / 2+2} c_{0} L_{n} x\right)\right)+\exp \left(-\frac{c(l)}{L_{n}^{2}}\right),
$$

где $c_{0}=\left(e^{1 / 2} / 6+\frac{1}{2 \sqrt{2}}+\frac{1}{2}\right) \approx 1.1285, c(l)=\frac{8}{27}\left(1-2^{-l}\right)^{3}$.

Заметим, что полученная оценка нетривиальна лишь при выполнении условия $x<\left(2^{3 l / 2+2} c_{0} L_{n}\right)^{-1}$. Если $X_{i}$ одинаково распределены, то это условие записывается следуюшим образом:

$$
x<\gamma_{0} \frac{\sigma^{3}}{\beta_{3}} \sqrt{n},
$$

где $\beta_{3}=\mathbf{E}\left|X_{1}\right|^{3}, \gamma_{0}=\left(2^{3 l / 2+2} c_{0}\right)^{-1}$.

С другой стороны, $\left|S_{n}\right| \leqslant \sqrt{n} V_{n}$, т.е. $\mathbf{P}\left\{S_{n}>x V_{n}\right\}=0$ для $x>\sqrt{n}$. Следовательно, оценка (2) не покрывает лишь зону между $\gamma_{0} \sigma^{3} \sqrt{n} / \beta_{3}$ и $\sqrt{n}$.

В неравенство (2) в отличие от соответствующего неравенства Нагаева-Фука, содержащего нормальную компоненту (см., например, [8]), не входит сумма вероятностей $\sum_{1}^{n} \mathrm{P}\left\{X_{i}>y\right\}$ и поправочный член $\left(A_{t} / x^{t}\right)^{x / y}$, где $A_{t}$ - сумма абсолютных моментов порядка $t$. По форме неравенство (2) наиболее близко к нижней оценке для вероятностей больших уклонений сумм независимых случайных величин, выведенной в статье [9].

Следуюший результат дополняет оценку (2).

Теорема 2. Пусть $\mathbf{E} X_{i}=0,1 \leqslant i \leqslant n, u C_{n}<\infty$. Тогда для $x \geqslant \gamma_{0} / L_{n} u$ любого натурального $l$

$$
\mathbf{P}\left\{S_{n}>x V_{n}\right\} \leqslant\left(\frac{\gamma_{0} x}{8 L_{n} \ln 2}+l+2\right) \exp \left(-\frac{\gamma_{0} x}{8 L_{n}}\right)+\exp \left(-\frac{c(l)}{L_{n}^{2}}\right),
$$

где второе слагаемое такое же, как и в неравенстве (2).

По форме неравенства (2) и (3) близки к неравенствам Бернштейна и Петрова (см., например, [10, гл. $3, \S 4$, теоремы 16 и 18]), однако, и это следует подчеркнуть особо, они не требуют никаких ограничений на моменты выше третьего.

Из неравенства (3) с очевидностью следует, что сушествует константа $c$ такая, что

$$
\mathbf{P}\left\{S_{n}>x V_{n}\right\}<\exp \left(-\frac{c}{L_{n}^{2}}\right)
$$

при $\gamma_{0} / L_{n} \leqslant x \leqslant \sqrt{n}$. Следуюший пример показывает, что в неравенстве (4) нельзя заменить $L_{n}^{-2}$ на $n$.

П р и м е р. Пусть $X, X_{1}, \ldots, X_{n}-$ независимые одинаково распределенные случайные величины, $\mathbf{P}\{X=1\}=1-p, \mathbf{P}\{X=(p-1) / p\}=p$. Оценим величину $\mathbf{P}\left\{S_{n}=\sqrt{n} V_{n}\right\}$. Несложно видеть, что

Заметим, что при $p<\frac{1}{2}$

$$
\mathbf{P}\left\{S_{n}=\sqrt{n} V_{n}\right\}=\mathbf{P}^{n}\{X=1\}=(1-p)^{n} .
$$

$$
(1-p)^{n}>\exp \left(-\frac{3}{2} n p\right)
$$


Подсчитаем теперь ляпуновское отношение $\mathbf{E}|X|^{3} /\left(\mathbf{E} X^{2}\right)^{3 / 2}$. Очевидно,

$$
\mathbf{E} X^{2}=\left(\frac{p-1}{p}\right)^{2} p+1-p=\frac{1-p}{p} .
$$

Далее, $\mathbf{E}|X|^{3}=((1-p) / p)^{3} p+1-p=(1-p) / p^{2}\left(1-2 p+2 p^{2}\right)$. Поэтому

$$
\frac{\mathbf{E}|X|^{3}}{\left(\mathbf{E} X^{2}\right)^{3 / 2}}=\frac{1-2 p+2 p^{2}}{\sqrt{p(1-p)}} \text {. }
$$

Следовательно,

$$
n p=L_{n}^{-2} \frac{\left(1-2 p+2 p^{2}\right)^{2}}{1-p}
$$

В результате имеем

$$
\mathbf{P}\left\{S_{n}=\sqrt{n} V_{n}\right\}>\exp \left(-\frac{c(p)}{L_{n}^{2}}\right),
$$

где $c(p)=3\left(1-2 p+2 p^{2}\right)^{2} /(2(1-p))$.

Конечно, возникает вопрос, насколько точны оценки (2) и (3). Ниже в виде небольшой таблицы приведены приближенные значения постоянных, участвующих в теоремах 1 и 2 , для значений $l=1,2,3$.

\section{Таблица 1.}

\begin{tabular}{|l|l|l|l|l|}
\hline$l$ & $c_{1}(l)$ & $c_{2}(l)$ & $c_{3}(l)$ & $\gamma_{0}(l)$ \\
\hline 1 & 12.765713 & 0.001236 & 0.009792 & 0.078335 \\
\hline 2 & 36.106889 & 0.008673 & 0.003462 & 0.027696 \\
\hline 3 & 102.125703 & 0.015687 & 0.001224 & 0.009792 \\
\hline
\end{tabular}

Здесь $c_{1}(l)=2^{3 l / 2+2} c_{0}, c_{3}(l)=\gamma_{0}(l) / 8$. В качестве $c_{2}(l)$ берется $c(l)$.

В таблице 2 приведены значения оценок (2) и (3) для $l=1$, вычисленные с помощью предыдущей таблицы. Значения выше разделительной линии соответствуют оценке (2), а ниже этой линии - оценке (3). Вычисления произведены с точностью до $10^{-6}$.

\section{Таблица 2.}

\begin{tabular}{|r|l|l|l|l|l|}
\hline$x \backslash L_{n}^{-2}$ & 3000 & 10000 & 15000 & 20000 & 25000 \\
\hline 1 & 2.774351 & 2.702184 & 2.686403 & 2.677039 & 2.670668 \\
2 & 2.605084 & 2.109839 & 2.013257 & 1.957800 & 1.920833 \\
3 & 3.175261 & 1.558555 & 1.330579 & 1.210873 & 1.135424 \\
4 & $\underline{6.692966}$ & 1.238906 & 0.851605 & 0.681067 & 0.584738 \\
5 & 0.470326 & 1.253775 & 0.602907 & 0.389676 & 0.289301 \\
6 & 0.306102 & 1.946563 & 0.549310 & 0.258392 & 0.154439 \\
7 & 0.197178 & $\underline{5.623631}$ & 0.754240 & 0.227715 & 0.100565 \\
8 & 0.125940 & 0.005672 & 1.830928 & 0.306398 & 0.090459 \\
9 & 0.079870 & 0.002341 & $\underline{9.219483}$ & 0.723213 & 0.127314 \\
10 & 0.050347 & 0.000959 & 0.000126 & 3.439368 & 0.317432 \\
11 & 0.031572 & 0.000390 & 0.000041 & $\underline{37.83}$ & 1.586743 \\
12 & 0.019709 & 0.000158 & 0.000013 & 0.000002 & $\underline{17.99}$ \\
13 & 0.012255 & 0.000063 & 0.000004 & 0.000000 & 0.000000 \\
14 & 0.007593 & 0.000025 & 0.000001 & 0.000000 & 0.000000 \\
15 & 0.004690 & 0.000010 & 0.000000 & 0.000000 & 0.000000 \\
16 & 0.002889 & 0.000004 & 0.000000 & 0.000000 & 0.000000 \\
17 & 0.001775 & 0.000002 & 0.000000 & 0.000000 & 0.000000 \\
18 & 0.001088 & 0.000001 & 0.000000 & 0.000000 & 0.000000 \\
19 & 0.000665 & 0.000000 & 0.000000 & 0.000000 & 0.000000 \\
20 & 0.000406 & 0.000000 & 0.000000 & 0.000000 & 0.000000 \\
\hline
\end{tabular}


Таблица 2 (продолжение).

\begin{tabular}{|r|l|l|l|l|}
\hline$x \backslash L_{n}^{-2}$ & 30000 & 40000 & 100000 & 1000000 \\
\hline 1 & 2.665975 & 2.659401 & 2.643847 & 2.625663 \\
2 & 1.893994 & 1.856952 & 1.771825 & 1.676647 \\
3 & 1.082763 & 1.012938 & 0.864572 & 0.717582 \\
4 & 0.522484 & 0.446118 & 0.306486 & 0.197051 \\
5 & 0.232202 & 0.170543 & 0.081922 & 0.034572 \\
6 & 0.105623 & 0.061966 & 0.017454 & 0.003931 \\
7 & 0.055009 & 0.023586 & 0.003154 & 0.000296 \\
8 & 0.036758 & 0.010384 & 0.000515 & 0.000015 \\
9 & 0.035319 & 0.005839 & 0.000081 & 0.000001 \\
10 & 0.054673 & 0.004629 & 0.000013 & 0.000000 \\
11 & 0.152681 & 0.005710 & 0.000002 & 0.000000 \\
12 & 0.860999 & 0.012083 & 0.000000 & 0.000000 \\
13 & $\underline{10.98}$ & 0.048362 & 0.000000 & 0.000000 \\
14 & 0.000000 & 0.403527 & 0.000000 & 0.000000 \\
15 & 0.000000 & $\underline{7.733759}$ & 0.000000 & 0.000000 \\
16 & 0.000000 & 0.000000 & 0.000000 & 0.000000 \\
17 & 0.000000 & 0.000000 & 0.000000 & 0.000000 \\
18 & 0.000000 & 0.000000 & 0.000000 & 0.000000 \\
19 & 0.000000 & 0.000000 & 0.000000 & 0.000000 \\
20 & 0.000000 & 0.000000 & 0.000001 & 0.000000 \\
\hline
\end{tabular}

Мы видим, что оценка (2) дает приемлемый результат только при очень малых значениях $L_{n}$. При больших значениях $x$ происходит стабилизация значений, поскольку основную роль начинает играть второе слагаемое оценки (3), которое не зависит от $x$.

Заметим, что первое слагаемое в правой части неравенства (2) не может быть меньше 0.66 ни при каких значениях $L_{n}$, если $x=3$.

Посмотрим, что получится, если убрать неприятный множитель перед экспонентой и заменить $x^{2} / 4$ на $x^{2} / 2$ и $2^{3 l / 2+2}$ на 1 . Тогда первое слагаемое в (2) не превосходит

$$
P\left(x, L_{n}\right) \equiv \exp \left\{-\frac{x^{2}}{2}\left(1-c_{0} L_{n} x\right)\right\} .
$$

При $x=3$ и $L_{n}<1 /\left(10 c_{0}\right)$ мы получаем оценку

$$
P\left(3, L_{n}\right) \leqslant \exp \left\{-\frac{81}{20}\right\} \approx 0.017 .
$$

По-видимому, постоянные в теоремах 1 и 2 далеки от оптимальных и их можно уточнить, применив более тонкую технику. Этот вопрос требует дальнейших исследований.

Интересно сравнить полученные нами оценки с неравенством (1). Во-первых, оценка (1) нетривиальна при $x>60.5$. Далее, для любого $\varepsilon>0$

$$
\begin{aligned}
\mathbf{P}\left\{\frac{S_{n}}{V_{n}}>x\left(4 \frac{B_{n}}{V_{n}}+1\right)\right\}= & \mathbf{P}\left\{\frac{S_{n}}{V_{n}}>x\left(4 \frac{B_{n}}{V_{n}}+1\right), V_{n}<\varepsilon B_{n}\right\} \\
& +\mathbf{P}\left\{\frac{S_{n}}{V_{n}}>x\left(4 \frac{B_{n}}{V_{n}}+1\right), V_{n} \geqslant \varepsilon B_{n}\right\}=P_{1}+P_{2} .
\end{aligned}
$$

Очевидно,

$$
P_{2}>\mathbf{P}\left\{\frac{S_{n}}{V_{n}}>x\left(\frac{4}{\varepsilon}+1\right), V_{n} \geqslant \varepsilon B_{n}\right\}>\mathbf{P}\left\{\frac{S_{n}}{V_{n}}>x\left(\frac{4}{\varepsilon}+1\right)\right\}-\mathbf{P}\left\{V_{n}<\varepsilon B_{n}\right\} .
$$

В результате мы получаем неравенство

$$
\mathbf{P}\left\{\frac{S_{n}}{V_{n}}>x\left(\frac{4}{\varepsilon}+1\right)\right\}<\mathbf{P}\left\{S_{n}>x\left(4 B_{n}+V_{n}\right)\right\}+\mathbf{P}\left\{V_{n}<\varepsilon B_{n}\right\} .
$$


Оценивая первое слагаемое в правой части с помощью (1), а второе с помощью леммы 3 , имеем для $0<\varepsilon<1$

$$
\mathbf{P}\left\{\frac{S_{n}}{V_{n}}>x\right\}<24 \exp \left(-\frac{x^{2} \varepsilon^{2}}{1152(4+\varepsilon)^{2}}\right)+\exp \left(-\frac{8\left(1-\varepsilon^{2}\right)^{3}}{27 L_{n}^{2}}\right) .
$$

По форме это неравенство близко к (2). Разница заключается в том, что в первом слагаемом в показателе экспоненты отсутствует ляпуновское отношение. Из таблицы 2 следует, что неравенство (2) может давать нетривиальный результат при $x \leqslant 4$. Правда при этом ляпуновское отношение должно быть не больше 0.008165 . В то же время неравенство (5) нетривиально лишь при $x>60.5(4 / \varepsilon+1)$.

Рассмотрим теперь $S_{n}^{2} / V_{n}^{2}$ как случайное блуждание. Положим

$$
\triangle_{n+1}=\frac{S_{n+1}^{2}}{V_{n+1}^{2}}-\frac{S_{n}^{2}}{V_{n}^{2}}
$$

Нетрудно видеть, что

$$
\triangle_{n+1}<\sup _{a, b, x}\left(\frac{(a+x)^{2}}{b^{2}+x^{2}}-\frac{a^{2}}{b^{2}}\right)
$$

Найдем

$$
\sup _{x} \frac{(a+x)^{2}}{b^{2}+x^{2}}
$$

для фиксированных $a$ и $b$.

Очевидно,

$$
\frac{d}{d x} \ln \frac{(a+x)^{2}}{b^{2}+x^{2}}=\frac{2}{a+x}-\frac{2 x}{b^{2}+x^{2}}=\frac{2\left(b^{2}-a x\right)}{(a+x)\left(b^{2}+x^{2}\right)} .
$$

Значит,

$$
\sup _{x} \frac{(a+x)^{2}}{b^{2}+x^{2}}=1+\frac{a^{2}}{b^{2}}
$$

Возврашаясь к (6), видим, что $\triangle_{n+1} \leqslant 1$.

Легко заметить, что

$$
\triangle_{n+1}=\frac{2 X_{n+1} S_{n}}{V_{n}^{2}}+\left(1-\frac{S_{n+1}^{2}}{V_{n+1}^{2}}\right) \frac{X_{n+1}^{2}}{V_{n}^{2}} \leqslant \frac{2 X_{n+1} S_{n}}{V_{n}^{2}}+\frac{X_{n+1}^{2}}{V_{n}^{2}} .
$$

Следовательно, случайное блуждание $S_{n}^{2} / V_{n}^{2}$ мажорируется некоторым субмартингалом.

\section{2. Вспомогательные утверждения.} $y>0$

Лемма 1. Для произвольных случайньх величин $X$ u $Y$ ивсех $h>0, x>0 u$

$$
\mathbf{P}\left\{X \geqslant x, Y^{2} \leqslant y^{2}\right\} \leqslant e^{h^{2} y^{2}-h x} \mathbf{E} e^{h X-h^{2} Y^{2}} .
$$

Д о к а з а т е л ь с т в о. Требуемая оценка следует из неравенства

$$
\mathbf{E}\left\{e^{h X-h^{2} Y^{2}} ; X \geqslant x, Y^{2} \leqslant y^{2}\right\} \geqslant e^{h x-h^{2} y^{2}} \mathbf{P}\left\{X \geqslant x, Y^{2} \leqslant y^{2}\right\} .
$$

Лемма 2. Для прозвольной случайной величины $X$ с $\mathbf{E} X=0$ и любого $h>0$

$$
\mathbf{E} e^{h X-h^{2} X^{2} / 2} \leqslant 1+c_{0} \beta_{3} h^{3}
$$

где $c_{0}=\left(e^{1 / 2} / 6+\frac{1}{2 \sqrt{2}}+\frac{1}{2}\right), \beta_{3}=\mathbf{E}|X|^{3}$.

Д о к а з а т е л ь с т в о. Раскладывая $e^{h X}$ в ряд Тейлора, имеем

$$
\begin{aligned}
\mathbf{E} \exp \left\{h X-\frac{h^{2} X^{2}}{2}\right\}= & \mathbf{E} e^{-h^{2} X^{2} / 2}+h \mathbf{E} X e^{-h^{2} X^{2} / 2}+\frac{h^{2}}{2} \mathbf{E} X^{2} e^{-h^{2} X^{2} / 2} \\
& +\mathbf{E}\left(e^{h X}-1-h X-\frac{h^{2} X^{2}}{2}\right) e^{-h^{2} X^{2} / 2}
\end{aligned}
$$


Очевидно,

$$
\mathbf{E} e^{-h^{2} X^{2} / 2}=1-\sigma^{2} \frac{h^{2}}{2}+\mathbf{E}\left(e^{-h^{2} X^{2} / 2}-1+\frac{h^{2} X^{2}}{2}\right),
$$

где $\sigma^{2}=\mathbf{E} X^{2}$. Далее,

$$
\begin{aligned}
\mathbf{E}\left(e^{-h^{2} X^{2} / 2}-1+\frac{h^{2} X^{2}}{2}\right)= & \mathbf{E}\left\{e^{-h^{2} X^{2} / 2}-1+\frac{h^{2} X^{2}}{2} ; \frac{h^{2} X^{2}}{2} \leqslant 1\right\} \\
& +\mathbf{E}\left\{e^{-h^{2} X^{2} / 2}-1+\frac{h^{2} X^{2}}{2} ; \frac{h^{2} X^{2}}{2}>1\right\} \equiv E_{1}+E_{2}
\end{aligned}
$$

Нетрудно видеть, что

$$
\begin{aligned}
& E_{1} \leqslant \frac{h^{4}}{8} \mathbf{E}\left\{X^{4} ; \frac{h^{2} X^{2}}{2} \leqslant 1\right\} \leqslant \frac{h^{3}}{4 \sqrt{2}} \mathbf{E}\left\{|X|^{3} ; \frac{h^{2} X^{2}}{2} \leqslant 1\right\}, \\
& E_{2} \leqslant \frac{h^{2}}{2} \mathbf{E}\left\{X^{2} ; \frac{h^{2} X^{2}}{2}>1\right\}<\frac{h^{3}}{2 \sqrt{2}} \mathbf{E}\left\{|X|^{3} ; \frac{h^{2} X^{2}}{2}>1\right\} .
\end{aligned}
$$

Следовательно,

$$
\mathbf{E}\left(e^{-h^{2} X^{2} / 2}-1+\frac{h^{2} X^{2}}{2}\right)<\frac{h^{3}}{2 \sqrt{2}} \beta_{3} .
$$

Из (10) и (11) следует, что

$$
\mathbf{E} e^{-h^{2} X^{2} / 2}=1-\frac{h^{2}}{2}+\theta \frac{h^{3}}{2 \sqrt{2}} \beta_{3}, \quad|\theta| \leqslant 1 .
$$

Нетрудно видеть, что

$$
\mathbf{E} X e^{-h^{2} X^{2} / 2}=\mathbf{E} X\left(e^{-h^{2} X^{2} / 2}-1\right)=\theta \frac{\beta_{3} h^{3}}{2} .
$$

Далее,

Если $x>0$, то

$$
\mathbf{E} X^{2} e^{-h X^{2}} \leqslant \sigma^{2}
$$

$$
e^{h x}-1-h x-\frac{h^{2} x^{2}}{2}<e^{h x} \frac{h^{3} x^{3}}{6} .
$$

Заметим, что $h x-h^{2} x^{2} / 2 \leqslant \frac{1}{2}$. Стало быть, для $x>0$

$$
\left(e^{h x}-1-h x-\frac{h^{2} x^{2}}{2}\right) e^{-h^{2} x^{2} / 2} \leqslant e^{h x-h^{2} x^{2} / 2} \frac{h^{3} x^{3}}{6} \leqslant \frac{e^{1 / 2}}{6} h^{3} x^{3} .
$$

Для $x \leqslant 0$

$$
\left(e^{h x}-1-h x-\frac{h^{2} x^{2}}{2}\right) e^{-h^{2} x^{2} / 2} \leqslant \frac{h^{3}|x|^{3}}{6} .
$$

Значит,

$$
\mathbf{E}\left(e^{h X}-1-h X-\frac{h^{2} X^{2}}{2}\right) e^{-h^{2} X^{2} / 2} \leqslant \frac{e^{1 / 2}}{6} h^{3} \beta_{3} .
$$

Комбинируя (9) и (12)-(15), получаем требуемый результат.

3 а м е ч а н и е. Неравенство типа (8) с постоянной $c_{0}=28.462$ можно получить из леммы 1 в работе [3].

Лемма 3. Для всех $0<\varepsilon<1$

$$
\mathbf{P}\left\{\sum_{1}^{n} X_{i}^{2}<B_{n}^{2} \varepsilon\right\} \leqslant \exp \left(-\frac{8(1-\varepsilon)^{3}}{27 L_{n}^{2}}\right) .
$$


Д о к а з а т ел ь с т в о. Нетрудно видеть, что для любой случайной величины $X$

$$
\mathbf{E} e^{-h X^{2}}=1-h b^{2}+\mathbf{E}\left(e^{-h X^{2}}-1+h X^{2}\right) .
$$

Очевидно,

$$
\begin{aligned}
\mathbf{E}\left(e^{-h X^{2}}-1+h X^{2}\right)= & \mathbf{E}\left(e^{-h X^{2}}-1+h X^{2} ; h X^{2} \leqslant \sqrt{2}\right) \\
& +\mathbf{E}\left(e^{-h X^{2}}-1+h X^{2} ; h X^{2}>\sqrt{2}\right)=E_{1}+E_{2} .
\end{aligned}
$$

Замечая, что

$$
\begin{aligned}
& E_{1}<\frac{h^{2}}{2} \mathbf{E}\left(X^{4} ; h X^{2} \leqslant \sqrt{2}\right) \leqslant \frac{h^{3 / 2}}{\sqrt{2}} \mathbf{E}\left(|X|^{3} ; h X^{2} \leqslant \sqrt{2}\right), \\
& E_{2}<h \mathbf{E}\left(X^{2} ; h X^{2}>\sqrt{2}\right) \leqslant \frac{h^{3 / 2}}{\sqrt{2}} \mathbf{E}\left(|X|^{3} ; h X^{2} \leqslant \sqrt{2}\right),
\end{aligned}
$$

мы приходим к неравенству

$$
\mathbf{E} e^{-h X^{2}} \leqslant 1-b^{2} h+\frac{\beta_{3}}{\sqrt{2}} h^{3 / 2} .
$$

Следовательно,

$$
\mathbf{P}\left\{\sum_{1}^{n} X_{i}^{2}<x\right\}<e^{h x} \prod_{1}^{n} \mathbf{E} e^{-h X_{i}^{2}}<\exp \left(\left(x-B_{n}^{2}\right) h+\frac{C_{n}}{\sqrt{2}} h^{3 / 2}\right) .
$$

При $x<B_{n}^{2}$

$$
\min _{h}\left(\left(x-B_{n}^{2}\right) h+\frac{C_{n}}{\sqrt{2}} h^{3 / 2}\right)
$$

достигается в точке $h_{0}$, удовлетворяюшей уравнению

$$
\left(x-B_{n}^{2}\right)+\frac{3}{2 \sqrt{2}} C_{n} h^{1 / 2}=0 .
$$

Очевидно,

$$
h_{0}^{1 / 2}=\frac{2 \sqrt{2}}{3} \frac{\overline{B_{n}^{2}}-x}{C_{n}} .
$$

В результате мы получаем оценку

$$
\mathbf{P}\left\{\sum_{1}^{n} X_{i}^{2}<x\right\}<\exp \left(-\frac{8\left(B_{n}^{2}-x\right)^{3}}{27 C_{n}^{2}}\right) .
$$

Полагая в этом неравенстве $x=\varepsilon B_{n}^{2}$, получаем желаемый результат.

3. Доказательство теоремы 1. Нетрудно видеть, что

$$
\begin{aligned}
\mathbf{P}\left\{S_{n}>V_{n} x ; y^{2} \leqslant V_{n}^{2}<2 y^{2}\right\} & \leqslant \mathbf{P}\left\{S_{n}>x y ; y^{2} \leqslant V_{n}^{2}<2 y^{2}\right\} \\
& \leqslant \mathbf{P}\left\{S_{n}>x y ; V_{n}^{2}<2 y^{2}\right\} .
\end{aligned}
$$

Полагая в лемме $1 X=S_{n}, Y^{2}=V_{n}^{2} / 2$, заключаем, что

$$
\mathbf{P}\left\{S_{n}>x y ; V_{n}^{2}<2 y^{2}\right\} \leqslant \exp \left(-h x y+y^{2} h^{2}\right) \mathbf{E} \exp \left(S_{n} h-\frac{V_{n}^{2} h^{2}}{2}\right) .
$$

Заметим, что $\min _{h}\left(y^{2} h^{2}-h x y\right)$ достигается при $h=x /(2 y)$.

Полагая $h=x /(2 y)$, имеем

$$
\min _{h}\left(y^{2} h^{2}-h x y\right)=-\frac{x^{2}}{4} .
$$

Применяя лемму 2 , получаем

$$
\mathrm{E} \exp \left(S_{n} h-\frac{V_{n}^{2} h^{2}}{2}\right)=\prod_{1}^{n} \mathbf{E} \exp \left(h X_{i}-\frac{h^{2} X_{i}^{2}}{2}\right)<\prod_{1}^{n}\left(1+c_{0} \beta_{3, i} h^{3}\right)<\exp \left(c_{0} C_{n} h^{3}\right) .
$$


Из (18)-(20) следует, что

$$
\mathbf{P}\left\{S_{n}>x y ; V_{n}^{2}<2 y^{2}\right\} \leqslant \exp \left(-\frac{x^{2}}{4}+c_{0} C_{n}\left(\frac{x}{2 y}\right)^{3}\right) .
$$

Очевидно,

$$
\begin{aligned}
\mathbf{P}\left\{S_{n}>V_{n} x ; V_{n}^{2} \geqslant 2^{-\imath} B_{n}^{2}\right\}= & \sum_{k=1-l}^{N} \mathbf{P}\left\{S_{n}>V_{n} x ; 2^{k-1} B_{n}^{2} \leqslant V_{n}^{2}<2^{k} B_{n}^{2}\right\} \\
& +\mathbf{P}\left\{S_{n}>V_{n} x ; V_{n}^{2} \geqslant 2^{N} B_{n}^{2}\right\} .
\end{aligned}
$$

Полагая $y^{2}=2^{k-1} B_{n}^{2}$ в (21), имеем

$$
\begin{aligned}
\mathbf{P}\left\{S_{n}>V_{n} x ; 2^{k-1} B_{n}^{2} \leqslant V_{n}^{2}<2^{k} B_{n}^{2}\right\} & \leqslant \mathbf{P}\left\{S_{n}>2^{(k-1) / 2} B_{n} x ; V_{n}^{2}<2^{k} B_{n}^{2}\right\} \\
& \leqslant \exp \left(-\frac{x^{2}}{4}+c_{0} C_{n}\left(\frac{x}{2^{(k-1) / 2} B_{n}}\right)^{3}\right) .
\end{aligned}
$$

По неравенству Маркова

$$
\mathbf{P}\left\{S_{n}>V_{n} x ; V_{n}^{2} \geqslant 2^{N} B_{n}^{2}\right\} \leqslant \mathbf{P}\left\{V_{n}^{2} \geqslant 2^{N} B_{n}^{2}\right\} \leqslant 2^{-N} .
$$

Пусть $N$ удовлетворяет условию

$$
2^{N} \leqslant e^{x^{2} / 4}<2^{N+1}
$$

Тогда

В результате имеем

$$
2^{-N}<2 e^{-x^{2} / 4}, \quad N \leqslant \frac{x^{2}}{4 \ln 2}
$$

$$
\mathbf{P}\left\{S_{n}>V_{n} x ; V_{n}^{2} \geqslant 2^{N} B_{n}^{2}\right\}<2 e^{-x^{2} / 4} .
$$

Из (22)-(25) следует, что

$$
\mathbf{P}\left\{S_{n}>V_{n} x ; V_{n}^{2}>2^{-l} B_{n}^{2}\right\}<\left(\frac{x^{2}}{4 \ln 2}+l\right) \exp \left(-\frac{x^{2}}{4}\left(1-2^{2+3 l / 2} c_{0} L_{n} x\right)\right) .
$$

Теперь рассмотрим случай $V_{n}^{2} \leqslant 2^{-l} B_{n}^{2}$. В силу леммы 3

$$
\mathbf{P}\left\{S_{n}>V_{n} x ; V_{n}^{2} \leqslant 2^{-l} B_{n}^{2}\right\}<\mathbf{P}\left\{V_{n}^{2} \leqslant 2^{-l} B_{n}^{2}\right\} \leqslant \exp \left\{-\frac{8}{27}\left(1-2^{-l}\right)^{3} L_{n}^{-2}\right\} .
$$

Комбинируя (26) и (27), получаем неравенство (2).

4. Доказательство теоремы 2. По (18) и (20)

$$
\mathbf{P}\left\{S_{n}>x y ; V_{n}<2 y^{2}\right\} \leqslant \exp \left(-h x y+y^{2} h^{2}+c_{0} C_{n} h^{3}\right) .
$$

Следовательно, полагая $y^{2}=2^{k-1} B_{n}^{2}$ и $h=\gamma_{0} /\left(2 y L_{n}\right)$, имеем

$$
\begin{aligned}
& \mathbf{P}\left\{S_{n}>x V_{n}, 2^{k-1} B_{n}^{2} \leqslant V_{n}^{2}<2^{k} B_{n}^{2}\right\} \leqslant \mathbf{P}\left\{S_{n}>x^{(k-1) / 2} B_{n} ; V_{n}^{2}<2^{k} B_{n}^{2}\right\} \\
& \quad \leqslant \exp \left(-\frac{x \gamma_{0}}{2 L_{n}}+\frac{\gamma_{0}^{2}}{4 L_{n}^{2}}+c_{0} C_{n}\left(\frac{\gamma_{0}}{2^{(k+1) / 2} L_{n} B_{n}}\right)^{3}\right) .
\end{aligned}
$$

Для $k \geqslant 1-l$

$$
\frac{c_{0} \gamma_{0}}{2^{3(k+1) / 2}}<\frac{1}{8}
$$

Следовательно,

$$
c_{0} C_{n}\left(\frac{\gamma_{0}}{2^{(k+1) / 2} L_{n} B_{n}}\right)^{3}<\frac{\gamma_{0}^{2}}{8 L_{n}^{2}} \text {. }
$$

Если $x>\gamma_{0} L_{n}^{-1}$, то

$$
x \gamma_{0} L_{n}^{-1}>\gamma_{0}^{2} L_{n}^{-2}
$$


Из (28)-(30) вытекает, что для $x>\gamma_{0} L_{n}^{-1}$

$$
\mathbf{P}\left\{S_{n}>x V_{n}, 2^{k-1} B_{n}^{2} \leqslant V_{n}^{2}<2^{k} B_{n}^{2}\right\} \leqslant \exp \left(-\frac{\gamma_{0} x}{8 L_{n}}\right) .
$$

Пусть $N$ таково, что

$$
2^{N} \leqslant \exp \left(\frac{\gamma_{0} x}{8 L_{n}}\right)<2^{N+1}
$$

Тогда

$$
\begin{aligned}
2^{-N-1} & <\exp \left(-\frac{\gamma_{0} x}{8 L_{n}}\right), \\
N & \leqslant \frac{\gamma_{0} x}{8 L_{n} \ln 2} .
\end{aligned}
$$

Используя (32) и неравенство Маркова, получаем

$$
\mathbf{P}\left\{S_{n}>x V_{n} ; V_{n}^{2}>2^{N} B_{n}\right\}<\mathbf{P}\left\{V_{n}^{2}>2^{N} B_{n}^{2}\right\}<2 \exp \left(-\frac{\gamma_{0} x}{8 L_{n}}\right) .
$$

С другой стороны, по (31) и (33)

$$
\begin{aligned}
\sum_{k=1-l}^{N} \mathbf{P}\left\{S_{n}>x V_{n} ; 2^{k-1} B_{n}^{2} \leqslant V_{n}^{2}<2^{k} B_{n}^{2}\right\} & <(N+l) \exp \left(-\frac{\gamma_{0} x}{8 L_{n}}\right) \\
& <\left(\frac{\gamma_{0} x}{8 L_{n} \ln 2}+l\right) \exp \left(-\frac{\gamma_{0} x}{8 L_{n}}\right) .
\end{aligned}
$$

Комбинируя (22), (27), (34), (35), приходим к оценке (3).

\section{СПИСОК ЛИТЕРАТУРЫ}

1. Logan B.F., Mallows C.L., Rice S.O., Shepp L.A. Limit distributions of selfnormalized sums. - Ann. Probab., 1973, v. 1, № 5, p. 788-809.

2. Bentkus V., Götze F. The Berry-Esseen bounds for Student's statistic. - Ann. Probab., 1996, v. 24, № 1, p. 491-503.

3. Shao Q.-M. A Cramér type large deviation result for Student's $t$-statistic. - J. Theoret. Probab., 1999, v. 12, № 2, p. 385-398.

4. Wang Q., Jing B.-Y. An exponential nonuniform Berry-Esseen bound for selfnormalized sums. - Ann. Probab., 1999, v. 27, № 4, p. 2068-2088.

5. Giné E., Götze F., Mason D. M. When is the Student $t$-statistic asymptotically standard normal? - Ann. Probab., 1997, v. 25, № 3, p. 1514-1531.

6. Giné E., Mason D. M. On the LIL for self-normalized sums of IID random variables. J. Theoret. Probab., 1998, v. 11, № 2, p. 351-370.

7. He X., Shao Q.-M. On parameters of increasing dimensions. - J. Multivariate Anal., 2000 , v. 73 , № 1 , p. $120-135$.

8. Нагаев С.В. Некоторые уточнения вероятностных и моментных неравенств. Теория вероятн. и ее примен., 1997, т. 42, в. 4, с. 832-838.

9. Нагаев C.B. Нижние оценки для вероятностей больших уклонений сумм независимых случайных величин. - Теория вероятн. и ее примен., 2001, т. 46, в. 1, c. $50-74$.

10. Петров В.В. Предельные теоремы для сумм независимых случайных величин. M.: Наука, 1987, 320 c. 